\title{
The Impact of CdO on the Radiation Shielding Properties of Zinc-Sodium-Phosphate Glass Containing Barium
}

\author{
A. Saleh ${ }^{a}$, R. M. El Shazly ${ }^{\text {, H.A. Abd El-Ghany }}{ }^{c, d}$ \\ ${ }^{(a)}$ Basic science Department, Higher technological institute, $10^{\text {th }}$ of Ramadan city, Egypt. \\ ${ }^{(b)}$ Physics Department, Faculty of Science, Al-Azhar University, Cairo, Egypt. \\ ${ }^{(c)}$ Department of Engineering Mathematics and Physics, Faculty of Engineering (Shoubra), Benha University, Cairo, Egypt. \\ ${ }^{(d)}$ Faculty of Sciences, Physics Department, Taibah University, Saudi Arabia.
}

\begin{tabular}{l} 
ART ICLE INFO \\
\hline Article history: \\
Received: $12^{\text {th }}$ June 2021 \\
Accepted: $9^{\text {th }}$ Oct. 2021 \\
\hline
\end{tabular}

Keywords:

Phosphate glasses,

Neutrons filter,

Gamma rays,

WINXCOM,

MCNP5.

\begin{abstract}
Glass series of chemical composition $40 \mathrm{P}_{2} \mathrm{O}_{5}-5 \mathrm{Na}_{2} \mathrm{O}-(35-x) \mathrm{ZnO}-x \mathrm{CdO}-20 \mathrm{BaO}$ where, $(x=0,7.5,15,22.5 \mathrm{~mol} . \%)$ have been prepared by the conventional melt-quenching technique. The shielding characteristics against gamma rays, fast, and slow neutrons have been investigated. The mass attenuation coefficient of the prepared glass samples for gamma rays $\left(\mu_{\mathrm{m}}\right)$ were determined experimentally at nine energy lines (ranged from 121.8 to $1407.9 \mathrm{keV}$ ) emitted from three different $\gamma$-rays' radioactive sources. The measured values of $\left(\mu_{m}\right)$ were compared with those estimated using MCNP5 code and calculated by WINXCOM software. A good agreement between both experimental and calculated values of $\mu_{\mathrm{m}}$ was observed. The half value layers (HVL), mean free path (MFP), effective atomic number $\left(Z_{\text {eff }}\right)$, effective electron density $\left(\mathrm{N}_{\mathrm{el}}\right)$, and radiation protection efficiency (RPE) were deduced. The results reveal the practicality of using the present glass composition as an effective agent for gamma-rays radioprotection. On the other hand, the macroscopic cross section $(\Sigma)$ of slow neutrons, total slow neutrons, and neutrons with energy $>10 \mathrm{keV}$ at different $\mathrm{CdO}$ contents have been determined under good experimental geometric condition. The results indicate a successive improvement of the shielding properties against fast and slow neutrons by increasing $\mathrm{CdO}$ concentrations. The effect of $\mathrm{CdO}$ addition is clear through the interaction of slow neutrons with cadmium nuclei inside the samples up to $15 \%$ which considered optimum $\mathrm{CdO}$ percentage in the glass barriers to be used as good neutrons filter.
\end{abstract}

\section{INTRODUCTION}

Presently, ionizing radiation is commonly used in numerous areas including medical therapy and diagnostics [1], nuclear power plants, reactor design, space technology applications, nuclear engineering [2], elemental analysis using non-destructive photoactivation analysis (PAA) [3, 4], metal-oxidesemiconductors (MOS) capacitor irradiation response test [5, 6], food treatment [7], archaeology (carbon dating) and security devices [8] etc. Long time exposure to radiation causes significant tissues damage including cancer, radiation infections, mutation, and sometimes even death. At genetic level, radiations further impact the future of living organ systems [9]. From this perspective, it is required to continue providing shielding materials to minimize exposure to radiation in areas where people are expected to receive ionizing radiation.

Almost every substance can be used to protect harmful radiation if this substance has sufficient thickness to absorb the radiation to a fair extent [10]. Commonly, different types of concretes are used to protect from harmful radiations [8]. There are many disadvantages of concrete such as destructive effects in its structure due to loss of water contained in it over the time of use and it's fully not transparence to visible light and thus it is impossible to look through a concrete radiation shield [11]. Because of their low cost, visible light transparence ability and easily to shape, glasses have increasingly studied by many researchers as promising radiation shielding materials [12]. 
Several studies have been performed on various glass systems to investigate their ability to attenuate different nuclear radiation. Most of these studies use lead as a former in these glassy structures. But due to its toxicity and high cost, lead however, may not be the optimum glassy former in the glass composition [13]. Already many studies to develop a new type of transparent glassy systems to enhance shielding properties have been devoted [14 -19]. As the ordinary window sand glasses based on silica and smaller amounts of alkalis oxides ( $\mathrm{Na} 2 \mathrm{O}$ or K2O) to reduce high melting point, many of the developed radiation shielding glasses are $\mathrm{SiO} 2-\mathrm{PbO}$ based. However, phosphate glasses are attractive for the design of many optical systems compared to other glass systems as the absorption edge of phosphate glasses is strongly shifted toward the short- wavelength range (next to UV range) making it more transparent for visible light [20].

Among other oxide glasses (borate, silicate, etc.), phosphate glasses have many unique advantages such as, ability to retain amorphous, it can handle highconcentration of transition metal ions [21, 22], unique chemical and physical structural and compositional capabilities that simplify modification of physical behavior and chemical substance [23]. Therefore, one or more multivalent oxides $(\mathrm{ZnO}, \mathrm{Al} 2 \mathrm{O} 3, \mathrm{SnO}, \mathrm{PbO}$, $\mathrm{Fe} 2 \mathrm{O} 3$, etc.), should be doped to develop the glass performance. These additions of such oxides help the development of the following chemical bonds (Zn-O-P, Al-O-P, Fe-O-P, Sn-O-P, Pb-O-P) and thus greatly increase their chemical stability [24]. In the present work $\mathrm{ZnO}$ was added. In addition, Ba-based glasses possess strong absorption for $\mathrm{x}$-ray and gamma-rays and non-toxicity compared with lead- and bismuth-based glasses [25]. Moreover, it has property to improve the rigidity of glass material [26].

From point of interest, $\mathrm{CdO}$ comes as a divalent metal oxide which can be featured with two different oxidation states. Because of its large atomic number, high density, low binding energy, $\mathrm{CdO}$ is considered here as an important oxide for different kinds radiation shielding applications. According to Osman AĞAR [27], addition of $\mathrm{CdO}$ as a network modifier enhances the shielding properties of tellurite-based glasses. Recently, the effect of adding $\mathrm{CdO}$ on the mechanical and radiation shielding properties of sodium borate glasses have been investigated [15].

Generally, In contrast to utilization of traditional shielding materials such as concrete, lead $(\mathrm{Pb})$, there is a continues need to obtain more glass compositions having some advantages such as: (i) possibility of transportation from one place to another, (ii) transparent materials that can be used in various technological applications such as iodine injection radiotherapy room. However, literature review showed that no study has been done on the radiation shielding properties of cadmium doped phosphate glasses. This has encouraged us to apply a broad-range investigation on the radiation shielding performance of different types of cadmium doped phosphate glass as a new member of the radiation shielding family.

The present work is devoted to study the effect of $\mathrm{CdO}$ on the shielding properties of zinc sodium phosphate glass containing barium. Hence, glass series of chemical composition $40 \mathrm{P}_{2} \mathrm{O}_{5}-5 \mathrm{Na}_{2} \mathrm{O}-(35-x) \mathrm{ZnO}$ $x$ CdO-20BaO where, $(x=0,7.5,15,22.5 \mathrm{~mol} . \%)$ have been prepared. So, the role of $\mathrm{CdO}$ shall be discussed for enhancing the shielding properties of the prepared glass against gamma rays, fast and slow neutrons in order to be used as a neutrons filter.

\section{Experimental and theoretical aspects}

\subsection{Samples preparation:}

Glasses in the $40 \mathrm{P}_{2} \mathrm{O}_{5}-5 \mathrm{Na}_{2} \mathrm{O}-(35-x) \mathrm{ZnO}-x \mathrm{CdO}-$ $20 \mathrm{BaO}(\mathrm{x}=0,7.5,15,22.5 \mathrm{~mol} \%)$ system have been prepared by the melt quenching method using high purity chemicals. After weighing and thoroughly mixing, batches of $90 \mathrm{~g}$ in weight were grinded in a mortar pestle for 30 minutes. The mixtures were heated for 30 minutes in a muffle furnace regulated at $260 \pm 5{ }^{\circ} \mathrm{C}$ to release the gases such as $\mathrm{CO}_{2}$ and $\mathrm{NH}_{3}$. Then the batches were melted in a platinum crucible with a closed lid at $1000 \pm 5{ }^{\circ} \mathrm{C}$ for $45 \mathrm{~min}$. Vitreous samples were obtained by pouring the melt into preheated stainless-steel molds. The samples then were annealed near the glass transition temperature for 2 hours to remove internal stresses. Finally, the glasses were cut, ground and polished to obtain samples with plan-parallel surfaces with dimensions $(4 \mathrm{~cm} \times 4 \mathrm{~cm})$ having an average thickness of $6 \mathrm{~mm}$ for further radiation shielding measurements. Density measurements of the synthesized glasses were achieved at room temperature using Archimedes' principle with toluene as an immersion liquid. a digital balance of sensitivity $10^{-4}$ $\mathrm{g}$ was used, and density values were obtained by three repeated measurements with an error of $\pm 0.05 \%$. The chemical composition (mole fraction) and density of prepared glass samples are listed in Table 1. 
Table (1): Chemical composition (mole fraction) and density of prepared glass samples.

\begin{tabular}{ccccccc}
\hline \multirow{2}{*}{ Sample } & \multicolumn{5}{c}{ Mole fraction } & $\begin{array}{c}\text { Density } \\
\left(\mathbf{g} / \mathbf{c m}^{\mathbf{3}}\right)\end{array}$ \\
\cline { 2 - 6 } & $\mathbf{P}_{2} \mathbf{O}_{5}$ & $\mathbf{Z n O}$ & $\mathbf{N a}_{2} \mathbf{O}$ & $\mathbf{C d O}$ & $\mathbf{B a O}$ & \\
\hline S1 & 0.4 & 0.35 & 0.05 & - & 0.2 & 3.015 \\
S2 & 0.4 & 0.275 & 0.05 & 0.075 & 0.2 & 3.349 \\
S3 & 0.4 & 0.200 & 0.05 & 0.150 & 0.2 & 3.458 \\
S4 & 0.4 & 0.125 & 0.05 & 0.225 & 0.2 & 3.498 \\
\hline
\end{tabular}

\subsection{Gamma ray measurements:}

The gamma ray attenuation coefficients of the prepared glass samples were obtained at nine energy lines using collimated beam of $\gamma$-rays emitted from 3.7 $\mu \mathrm{Ci}$ Eu-152, $9.5 \mu \mathrm{Ci} \mathrm{Cs}-137$, and $4.9 \mu \mathrm{Ci} \mathrm{Co}-60$ radioactive sources. Figure (1) shows the schematic diagram of gamma ray detection system, where a $3^{\prime \prime} \times 3$ " $\mathrm{NaI}$ (Tl) scintillation detector was used to measure the gamma ray intensities for the studied energy lines.

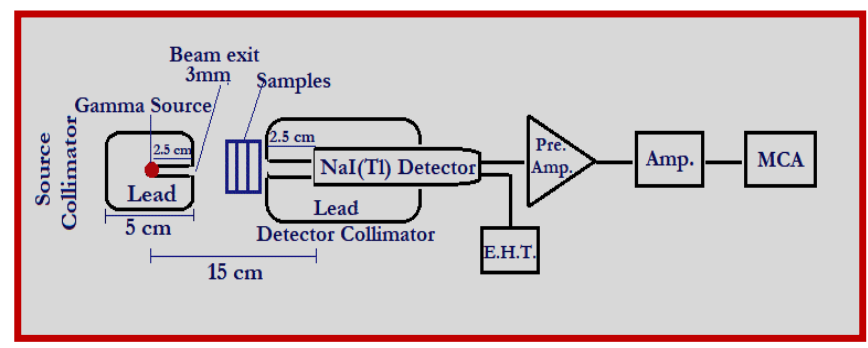

Fig. (1): Schematic diagram of gamma ray detection setup.

The capability of any absorber to attenuate the photons of certain energies can be estimated using the mass attenuation coefficient, $\mu_{m}\left(\mathrm{~cm}^{2} / \mathrm{g}\right)$ given by the Beer-Lambert's equation [28]:

$$
\mu_{m}=-\frac{\ln \left(\frac{I_{0}}{I}\right)}{x \rho}
$$

where $I_{0}$ and $I$ are the incident and the transmitted intensities, $\rho\left(\mathrm{g} / \mathrm{cm}^{3}\right)$ is the density of the material and $\mathrm{x}$ $(\mathrm{cm})$ is the thickness of the absorber.

The result values of $\mu_{\mathrm{m}}$ were compared by calculated values for a mixture of different elements by "WinXCom" computer program (version 3.1) [29]. And also, with that estimated by MCNP5 code.

$$
\mu_{m}=\sum_{i}^{n} W_{i}\left(\frac{\mu_{i}}{\rho_{i}}\right)
$$

where, $\left(\frac{\mu_{i}}{\rho_{i}}\right)$ is the mass attenuation coefficient for the individual element in each mixture sample, while $W_{i}$ is the fractional weight of the element in each mixture sample.

The mean free path, $\lambda(\mathrm{cm})$, the half value layer HVL $(\mathrm{cm})$, and tenth value layer TVL $(\mathrm{cm})$ of each sample concentration and total gamma rays, are characterize the effectiveness of gamma-ray shielding, were determined using the following equations [28]:

$\lambda=\frac{1}{\mu}, \quad H V L=\frac{\ln (2)}{\mu}, \quad T V L=\frac{\ln (10)}{\mu}$

where $\mu\left(\mathrm{cm}^{-1}\right)$ is the linear attenuation coefficient for the material under consideration.

The effective atomic number $Z_{\text {eff }}$ (dimensionless quantity) can be calculated by [28]:

$Z_{e f f}=\frac{\sigma_{t, a}}{\sigma_{t, e l}}=\frac{\sum_{i} n_{i} A_{i}(\mu / \rho)_{i}}{\sum_{i} n_{i} A_{i} / Z_{i}(\mu / \rho)_{i}}$

Electron density, $\mathrm{N}_{\mathrm{el}}$ (number of electron per gram) of the material can be derived using $\mu_{\mathrm{m}}$ and $\sigma_{\mathrm{t}, \mathrm{a}}$. [30]:

$N_{e l}=\frac{\mu_{m}}{\sigma_{e}}=\frac{Z_{e f f} N_{A} \sum_{i} n_{i}}{M}$

The radiation protection efficiency of a shielding material is defined as [31]:

$$
R P=\left(1-\frac{I}{I_{0}}\right) \times 100
$$

\subsection{Neutron measurements:}

The Boron tri-fluoride $\left(\mathrm{BF}_{3}\right)$ neutron detector was used to detect a collimated slow neutrons, total slow neutrons (primary slow as well as slowdown in the studied glass samples), and neutrons with energy greater than $10 \mathrm{keV}$ beam emitted from ${ }^{241} \mathrm{Am}-\mathrm{Be}$ neutron source with activity $3.7 \mathrm{GBq}$. The neutron transmitted fluxes were measured to deduce the values of macroscopic neutron cross-section $\left(\sum\right)$ according to equation (7) [32]. In case of slow neutrons measurements, the collimated beam was slowed down by $7 \mathrm{~cm}$ polyethylene block behind the sample, also the neutron of energy below $10 \mathrm{keV}$ was cut off by a block of Born carbide $\mathrm{B}_{4} \mathrm{C}$. The Schematic diagram of experimental setup was shown in Fig. 2.

$I_{n}=I_{o} e^{-\Sigma x}$

Where $\Sigma$ is the neutron macroscopic cross section $\left(\mathrm{cm}^{-1}\right)$, $I_{n}$ and $I_{o}$ are the neutron fluxes after and before transmitted through the sample barriers respectively, and $x$ is the sample thickness. 


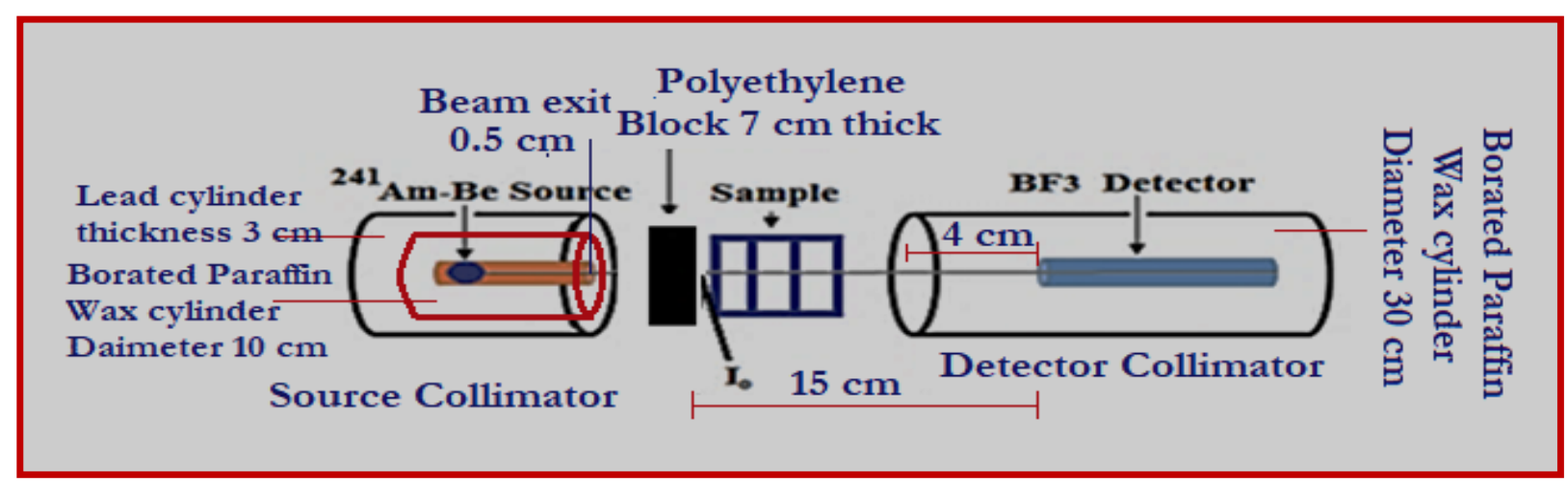

Fig. (2): Schematic diagram of Neutron detection setup.

The maximum experimental errors in attenuation coefficients and half value layers for both gamma-ray and neutron attenuation measurements were evaluated using the following error formulas [33].

$\Delta \sigma=\frac{1}{x \rho} \sqrt{\left(\frac{\Delta I_{0}}{I_{0}}\right)^{2}+\left(\frac{\Delta I_{(\gamma)}}{I_{(\gamma)}}\right)^{2}+\left(\frac{\Delta x \rho}{x \rho}\right)^{2}\left(\ln \left(\frac{I_{0}}{I_{(\gamma)}}\right)\right)^{2}}$

$(\Delta H V L)=(H V L) \sqrt{\left(\frac{\Delta \mu}{\mu}\right)^{2}(\ln 2)^{2}}$

$\Delta \Sigma=\frac{1}{x} \sqrt{\left(\frac{\Delta I_{0}}{I_{0}}\right)^{2}+\left(\frac{\Delta I_{(n)}}{I_{(n)}}\right)^{2}+\left(\frac{\Delta x}{x}\right)^{2}\left(\ln \left(\frac{I_{0}}{I_{(n)}}\right)\right)^{2}}$

$(\Delta M F P)=(M F P) \sqrt{\left(\frac{\Delta \Sigma}{\Sigma}\right)^{2}}$

\section{RESULTS AND DISCUSSION}

\subsection{X-ray diffraction studies}

$\mathrm{X}$-ray diffraction (XRD) technique was employed to check the glass formability of the obtained samples. Fig. 3 shows the XRD patterns of the prepared samples with different concentrations of $\mathrm{CdO}$. The results show diffused scattering with no Bragg's peaks can be observed. This confirms the amorphous nature of the prepared samples. One can observe a broad profile around $2 \theta=20^{\circ}$ which suggest the existence of some short-range order in the glass samples.

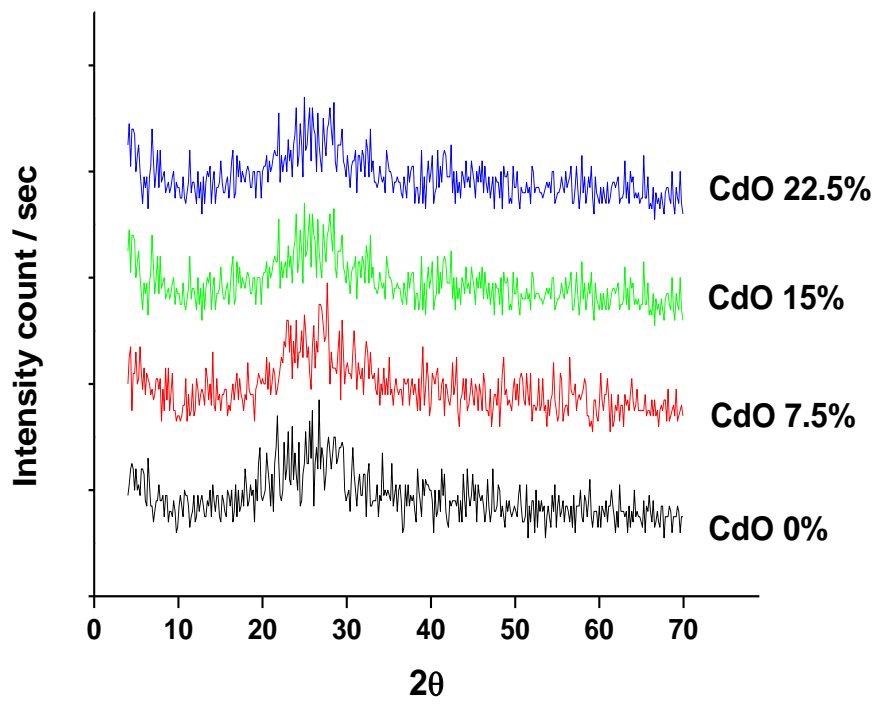

Fig. (3): XRD patterns of glass samples for different CdO content.

\subsection{Gamma-rays attenuation coefficients}

The attenuation coefficient $\left(\mu_{\mathrm{m}}\right)$ for the present glass samples have been determined at nine energy lines of gamma-rays $(121.78,344.27,661.64,778.9,964$, 1112.4, 1173.23, 1332.51, and $1407.92 \mathrm{keV})$. Different thicknesses of the glass barriers have been applied at each concentration of $\mathrm{CdO}$ (varying from 0 - 1.7 $\mathrm{cm})$. The measured $\gamma$-rays intensities transmitted through glass samples were given as a function of superficial densities $\rho_{x}\left(\mathrm{~g} / \mathrm{cm}^{3}\right)$ of glass barriers. The average values of the total mass attenuation coefficients $\mu_{\mathrm{m}}\left(\mathrm{cm}^{2} / \mathrm{g}\right)$ of the present glass samples were deduced using the slope of the attenuation curves. The measured values of mass attenuation coefficient $\left(\mu_{\mathrm{m}}\right)$ for the glass samples at different cadmium concentration have been compared with those estimated using MCNP5 code and those calculated using WINXCOM software and listed in table2. A quick look into the results in table 2 show a good agreement between the experimental values of 
the mass attenuation coefficient and the corresponding theoretical data calculated using WINXCOM program (version 3.1) and that estimated using MCNP5 code. However, the experimental values of the mass attenuation coefficient are found to be slightly lowered than those theoretically obtained from WINXCOM and MCNP5. This may be attributed to the crystalline nature of the molecular arrangement of detector source which can make incident beam geometry narrow, possibly reducing the $\mu_{\mathrm{m}}$ values[19].

Table (2): The experimentally measured mass attenuation coefficients $\left(\mathrm{cm}^{2} / \mathrm{g}\right)$ along with that estimated theoretically for the selected glasses.

\begin{tabular}{|c|c|c|c|c|c|c|c|c|c|c|c|c|}
\hline \multirow{2}{*}{$\begin{array}{c}\text { Energy } \\
(\mathrm{keV})\end{array}$} & \multicolumn{3}{|c|}{$\mathrm{S} 1$} & \multicolumn{3}{|c|}{$\mathrm{S} 2$} & \multicolumn{3}{|c|}{$\mathrm{S} 3$} & \multicolumn{3}{|c|}{ S4 } \\
\hline & WinX & Exp. & MCNP5 & WinX & Exp. & MCNP5 & WinX. & Exp. & MCNP5 & WinX. & Exp. & MCNP5 \\
\hline 121.78 & 0.383 & $0.3296 \pm 0.014$ & 0.3841 & 0.421 & $0.3729 \pm 0.004$ & 0.4217 & 0.458 & $0.3877 \pm 0.003$ & 0.4589 & 0.495 & $0.4448 \pm 0.004$ & 0.4920 \\
\hline 344.27 & 0.107 & $0.1104 \pm 0.016$ & 0.1081 & 0.109 & $0.1094 \pm 0.009$ & 0.1100 & 0.110 & $0.0911 \pm 0.003$ & 0.1111 & 0.112 & $0.0993 \pm 0.005$ & 0.1117 \\
\hline 661.64 & 0.075 & $0.0718 \pm 0.004$ & 0.0749 & 0.075 & $0.0722 \pm 0.003$ & 0.0753 & 0.075 & $0.0631 \pm 0.002$ & 0.0755 & 0.075 & $0.0638 \pm 0.002$ & 0.0752 \\
\hline 778.90 & 0.068 & $0.0682 \pm 0.003$ & 0.0691 & 0.069 & $0.0668 \pm 0.001$ & 0.0694 & 0.069 & $0.0609 \pm 0.001$ & 0.0683 & 0.068 & $0.0577 \pm 0.002$ & 0.0681 \\
\hline 964.00 & 0.062 & $0.0594 \pm 0.007$ & 0.0629 & 0.062 & $0.0579 \pm 0.005$ & 0.0617 & 0.062 & $0.0558 \pm 0.004$ & 0.0621 & 0.061 & $0.0532 \pm 0.006$ & 0.0621 \\
\hline 1112.4 & 0.057 & $0.0576 \pm 0.001$ & 0.0587 & 0.057 & $0.0569 \pm 0.001$ & 0.0581 & 0.057 & $0.0583 \pm 0.001$ & 0.0580 & 0.057 & $0.0507 \pm 0.001$ & 0.0579 \\
\hline 1173.2 & 0.055 & $0.0540 \pm 0.001$ & 0.0561 & 0.056 & $0.0529 \pm 0.001$ & 0.0561 & 0.056 & $0.0568 \pm 0.002$ & 0.0560 & 0.055 & $0.0470 \pm 0.001$ & 0.0556 \\
\hline 1333.5 & 0.052 & $0.0499 \pm 0.001$ & 0.0529 & 0.052 & $0.0498 \pm 0.001$ & 0.0528 & 0.052 & $0.0503 \pm 0.001$ & 0.0526 & 0.052 & $0.0431 \pm 0.001$ & 0.0528 \\
\hline 1407.9 & 0.050 & $0.0497 \pm 0.001$ & 0.0514 & 0.051 & $0.0487 \pm 0.003$ & 0.0520 & 0.051 & $0.0529 \pm 0.001$ & 0.0517 & 0.051 & $0.0439 \pm 0.001$ & 0.0515 \\
\hline
\end{tabular}

The energy dependence of the mass attenuation coefficient ( $\mu_{\exp }$ and $\left.\mu_{\text {WINXCOM }}\right)$ for different cadmium concentrations is depicted in Fig. 4.
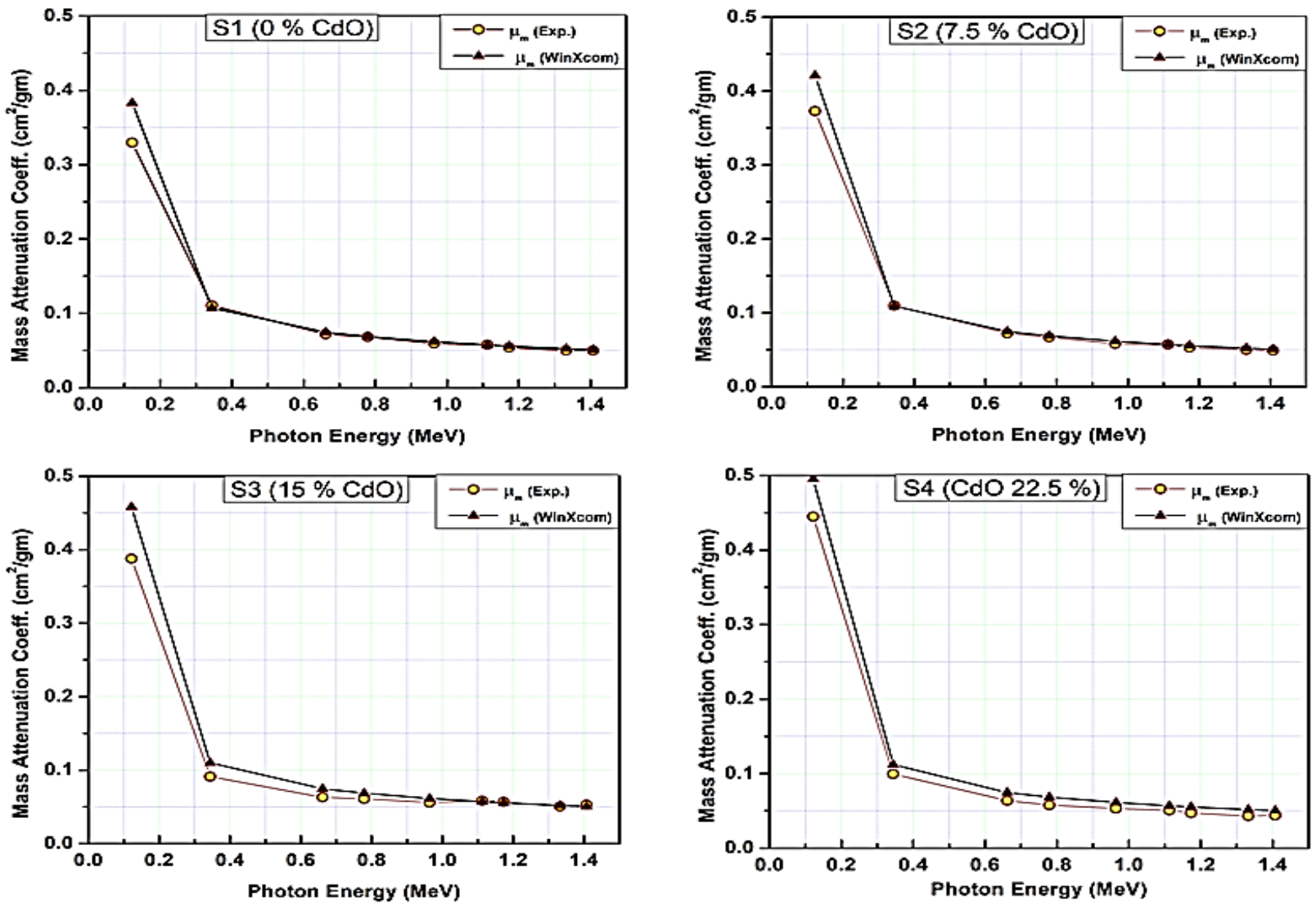

Fig. (4): Mass attenuation coefficient of the glass samples as a function of gamma ray energy. 
In general, both $\mu_{\exp }$ and $\mu_{\text {winXCOM }}$ follow one common trend for all glass samples where it decreases by increasing the photon energy. It is quite clear that at low photon energies (between 121 and $344 \mathrm{keV}$ ), the mass attenuation coefficient is found to be decreased dramatically with increasing the photon energy. This may be explained on the basis that the prominent reaction here is the photoelectric absorption (PE) mechanism [34]. Meanwhile in region from $344 \mathrm{keV}$ up to $1407 \mathrm{keV}$, a slight decrease in mass attenuation coefficient can be observed as the gamma-ray energy increases. Such behavior of the mass attenuation coefficient in this energy interval can be ascribed to the preeminence of Compton scattering process [35].

Fig. 5 shows the measured values of mass attenuation coefficient $\left(\mu_{\mathrm{m}}\right)$ for glass samples at different energies. It can be indicated that the mass attenuation coefficient for the present glass system is enhanced by increasing $\mathrm{CdO}$ content.

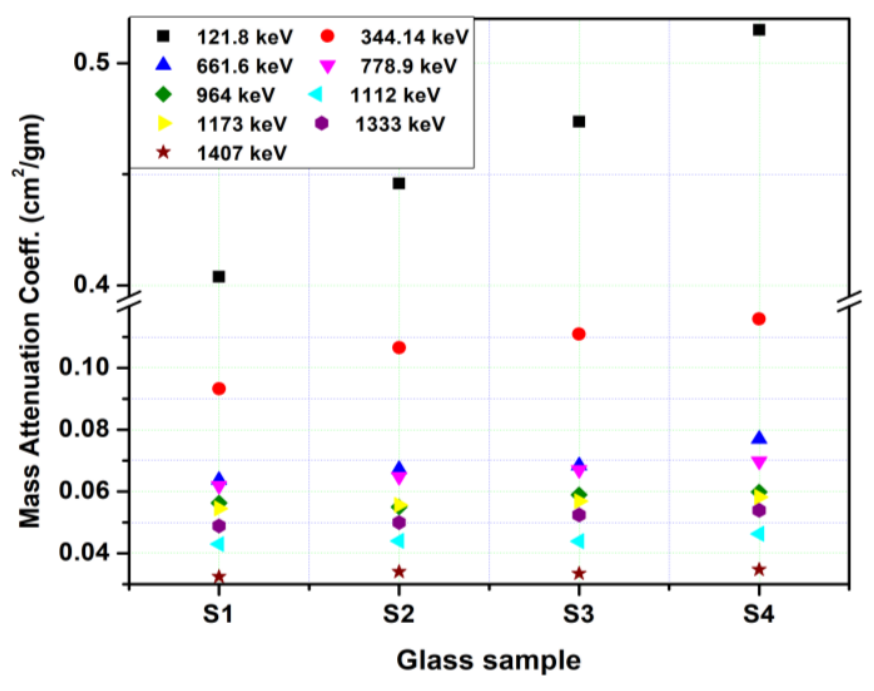

Fig. (5): The measured values of mass attenuation coefficient $\left(\mu_{\mathrm{m}}\right)$ for the glass samples at different energies.

The obtained results of $\mu / \rho$ were used to calculate the mean free path of prepared samples using eq.(3) and graphically introduced in Fig.6. It was observed that the values of MFP were reduced with the increase in $\mathrm{CdO}$ concentration as well as the density of glass samples (as can be seen in Table 1). From Fig.6, the MFP of glass sample (S4) possesses the lowest values. This indicates that, the radiation shielding performances of the present glasses are improved with the addition of $\mathrm{CdO}$ modifier.

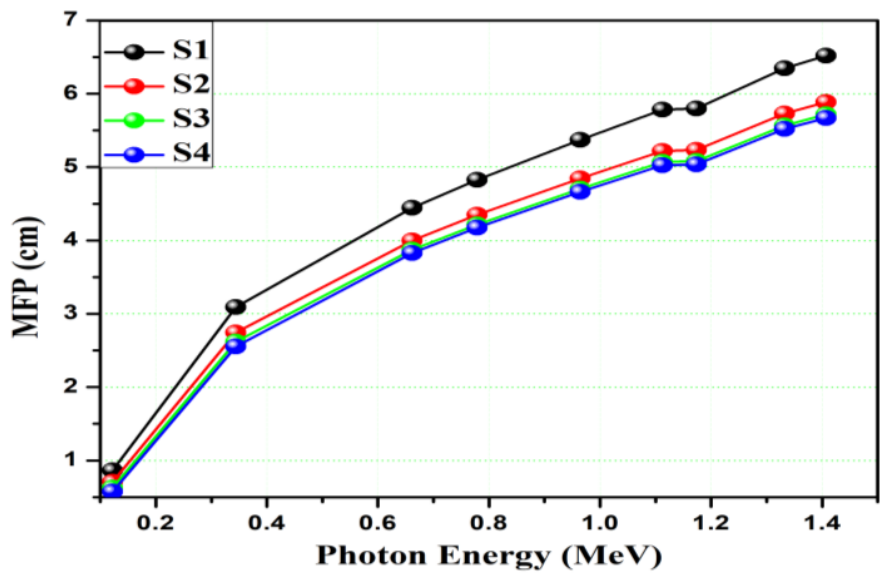

Fig. (6): Variation of mean free path with photon energy between 0.015 and $10 \mathrm{MeV}$.

The half value layer (HVL) for the present glass barrier samples is determined using equation (3). Table 3 shows the calculated values of HVL for the nine gamma-ray energies at different $\mathrm{CdO}$ concentrations. The obtained data reveal a general decrease in HVL for all energy lines by increasing content of $\mathrm{CdO}$. The percentage of decrement in HVL is found to be ranged from $50 \%$ (for $121.8 \mathrm{keV}$ ) to $15 \%$ (for $1407 \mathrm{KeV}$ ). In other words, HVL decreases more effectively for lower photon energies than for higher photon energies with increasing concentrations of $\mathrm{CdO}$ from $0 \%$ up to $22.5 \%$.

Table (3): Half value layer (HVL) for all samples.

\begin{tabular}{|c|c|c|c|c|c|}
\hline \multirow{2}{*}{$\begin{array}{c}\text { Energy } \\
(\mathrm{keV})\end{array}$} & \multicolumn{4}{|c|}{ HVL (cm) } & \multirow{2}{*}{$\begin{array}{c}\left(\text { HVL }_{S 1^{-}}\right. \\
\left.\text {HVL }_{S 4} / \text { HVL }_{S 1}\right) \\
100(\%)\end{array}$} \\
\hline & S1 & $\mathbf{S 2}$ & $\mathbf{S 3}$ & S4 & \\
\hline 121.78 & 0.5985 & 0.4911 & 0.4370 & 0.3995 & 49.8244 \\
\hline 344.27 & 2.1419 & 1.9013 & 1.8160 & 1.7708 & 20.9601 \\
\hline 661.64 & 3.0801 & 2.7723 & 2.6842 & 2.6529 & 16.1070 \\
\hline 778.90 & 3.3448 & 3.0142 & 2.9221 & 2.8914 & 15.6820 \\
\hline 964.00 & 3.7226 & 3.3589 & 3.2603 & 3.2302 & 15.2458 \\
\hline 1112.40 & 4.0076 & 3.6178 & 3.5134 & 3.4827 & 15.0734 \\
\hline 1173.23 & 4.0181 & 3.6269 & 3.5219 & 3.4908 & 15.1076 \\
\hline 1333.51 & 4.3981 & 3.9712 & 3.8574 & 3.8246 & 14.9955 \\
\hline 1407.92 & 4.5179 & 4.0797 & 3.9630 & 3.9295 & 14.9749 \\
\hline
\end{tabular}


The measured values of the mass attenuation coefficient have been utilized to evaluate the effective atomic number $\left(\mathrm{Z}_{\mathrm{eff}}\right)$ and electronic density $\left(\mathrm{N}_{\mathrm{el}}\right)$ for the present glass samples using Eqs. (4 \& 5) respectively. Figs. 7 and fig. 8 show that the variations of the calculated values of $Z_{\text {eff }}$ and $N_{\text {el }}$ as a function of $\gamma$-ray energies. It's clear that they have the same trend for all glass samples studied.

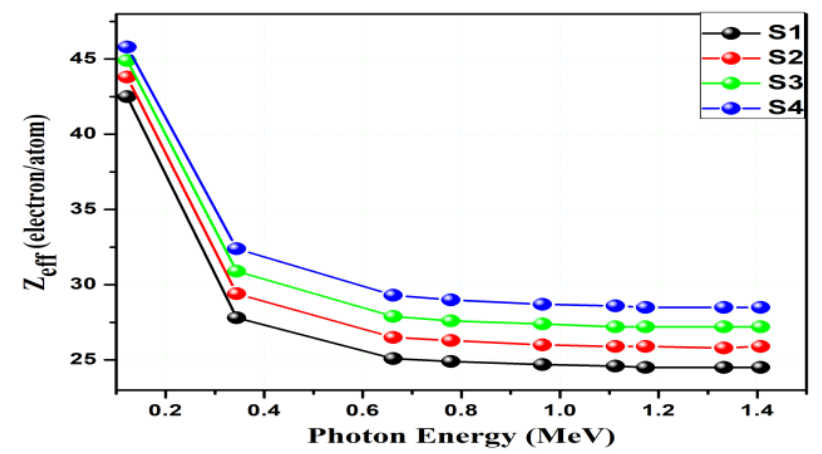

Fig. (7): Variation of $Z_{\text {eff }}$ with photon energy for all selected glass samples

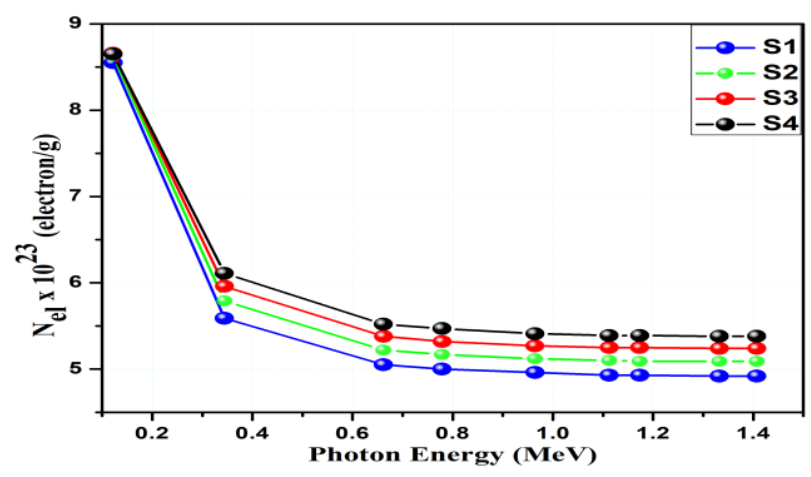

Fig. (8): Variation of $\mathrm{N}_{\mathrm{el}}$ with photon energy for all selected glass samples

In addition, figs. 7 and fig. 8 show that, the values of $Z_{\text {eff }}$ and $\mathrm{N}_{\mathrm{el}}$ were decreased for all glass samples with increasing the photon energy up to $0.356 \mathrm{MeV}$ (the photoelectric effect is dominant at low energies and hence $Z_{\text {eff }}$ is described by this process for the photon absorption). After that, they seem to be constant due to Compton scattering's linear $\mathrm{Z}$ dependency (Compton scattering is the most powerful process at this energy region). Here, both Compton scattering and pair production will be the main processes in comparison with photoelectric effect process and this can affect the photon absorption by $Z_{\text {eff. }}$.

It is very clear that for the prepared glass samples, the calculated values of $\mathrm{Z}_{\mathrm{eff}}$ and $\mathrm{N}_{\mathrm{el}}$ are improved by increasing the content of $\mathrm{CdO}$. As previously discussed, this can be due to changes in the mass attenuation coefficient. On the other hand, Fig. 9 reveals a strong correlation between $\mathrm{Z}_{\mathrm{eff}}$ and $\mathrm{N}_{\mathrm{el}}$ derived from the deduced values which verified the closely related photon energy dependence of $\mathrm{Z}_{\mathrm{eff}}$ and $\mathrm{N}_{\mathrm{el}}$.

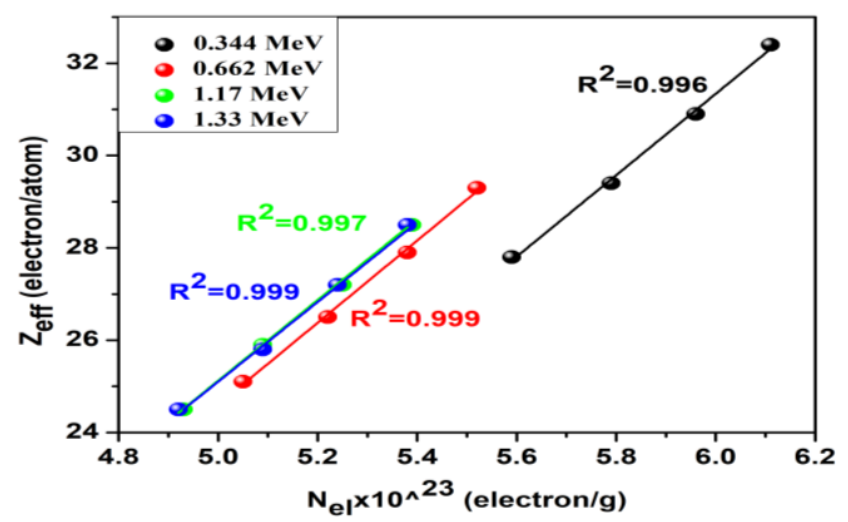

Fig. (9): Correlation between effective atomic number $\left(Z_{\text {eff }}\right)$ and electron density $\left(\mathrm{N}_{\mathrm{el}}\right)$ of glass samples for experimental results at $0.356,0.662,1.173$ and 1.33 MeV.

The variation of the RPE (radiation protection coefficient) as a function of photon energies for the present glasses have been determined and enlisted in Table. 4. The obtained data indicate that RPE reduces from its maximum value at $121 \mathrm{keV}$ with increasing the photon energy for all prepared glass samples. It can be clearly observed that the attenuation capability of gamma photons is increased by increasing $\mathrm{CdO}$ concentrations from $0 \%$ up to $22.5 \%$. The results reveal the practicality of using the present glass composition as an effective agent for gamma-rays shielding.

Table (4): Variation of experimentally measured RPE for the selected glasses with photon energy.

\begin{tabular}{lllll}
\hline \multirow{2}{*}{$\begin{array}{l}\text { Energy } \\
(\mathbf{k e V})\end{array}$} & $\mathbf{4}$ RPE $(\%)$ \\
\cline { 2 - 5 } & $\mathbf{S 1}$ & $\mathbf{S 2}$ & $\mathbf{S 3}$ & $\mathbf{S 4}$ \\
\hline 121.78 & 96.899 & 98.549 & 99.142 & 99.451 \\
344.27 & 62.115 & 66.494 & 68.172 & 69.089 \\
661.64 & 49.083 & 52.760 & 53.908 & 54.328 \\
778.90 & 46.289 & 49.829 & 50.909 & 51.277 \\
964.00 & 42.792 & 46.149 & 47.148 & 47.461 \\
1112.40 & 40.474 & 43.710 & 44.664 & 44.951 \\
1173.23 & 40.394 & 43.629 & 44.584 & 44.875 \\
1333.51 & 37.669 & 40.757 & 41.665 & 41.934 \\
1407.92 & 36.882 & 39.926 & 40.821 & 41.085 \\
\hline
\end{tabular}




\subsection{Neutron measurements and calculations.}

The measured neutron intensities transmitted through glass samples were given as a function of glass thickness (attenuation curves).The slope of these curves has been used to determine the values of macroscopic crosssections of slow neutrons, total slow neutrons and neutrons of energy $>10 \mathrm{keV}$ in the investigated glass samples. Fig.10 shows the variation of such values of the macroscopic cross-section with increasing concentrations of $\mathrm{CdO}$ in the samples. It can be observed that in case of slow and total slow neutrons, the values of macroscopic cross-section is increased by increasing content of $\mathrm{CdO}$ from $0 \%$ up to $15 \%$ and there is no significant increase in that values by increasing the concentration of $\mathrm{CdO}$ content above $15 \%$. In case of neutrons of energy $>10 \mathrm{keV}$, the values of macroscopic cross-section seem to be nearly constant by addition $\mathrm{CdO}$ to glass samples from 0 up to $22.5 \%$. Also, the data indicate that, the experimental values of $\sum$ for slow neutrons are the highest value from the others after addition of $\mathrm{CdO}$ in the samples. This can be explained by the highest probable interaction is a captured of slow neutron by the $\mathrm{Cd}$ added in glass samples; $(\mathrm{n}, \gamma)$ reactions, where the cross-section value of slow neutron with natural cadmium is about 2918 barn [43]. While for total slow neutrons there is a competition between two process in this range of neutron energies which considered slow down neutron energy due to inelastic scattering, i.e. $(\mathrm{n}, \mathrm{n})$ reactions for fast neutrons by the barium nuclei, and the cut-off neutron with energy less than $0.4 \mathrm{eV}$ by cadmium nuclei. But in the case of neutron with energies $>10 \mathrm{keV}$ there is no effect of cadmium nuclei on these similar neutron energies, so we found that there is no significant changes of the value of macroscopic cross-section by increasing the concentration of cadmium nuclei in glass sample, and Interaction values are attributed to inelastic scattering for neutrons by barium nuclei only.

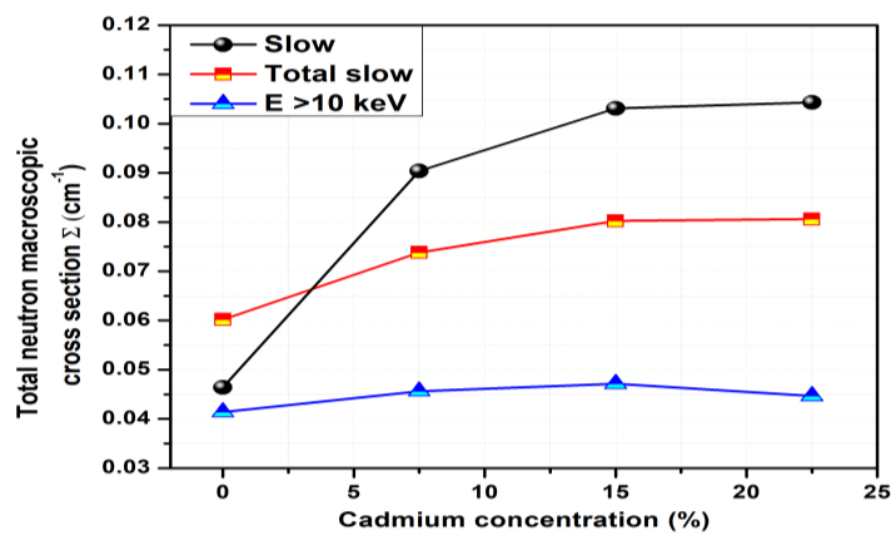

Fig. (10): Variation of total neutron, emitted from 241Am-Be source, macroscopic cross-section with $\mathrm{Cd}$ concentration in glass samples.
Fig. 11 shows the variation of the total neutron crosssection with neutron energy for cadmium and zinc as estimated using MCNP5 code. It can be easily observed that cadmium have a total neutron cross-section larger than that of zinc especially in the low energy range.

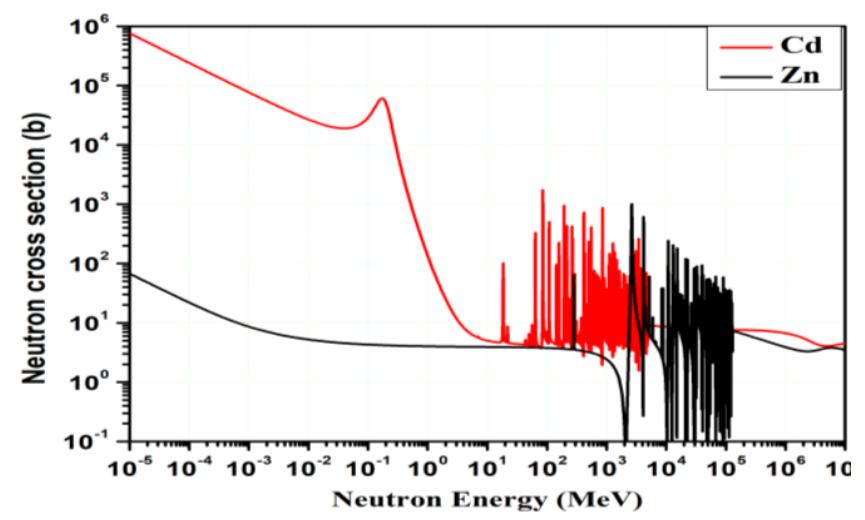

Fig. (11): Variation of total neutron cross-section with neutron energy of $\mathrm{Cd}$ and $\mathrm{Zn}$.

In addition, some important parameters of neutron attenuation have been determined like half value layer (HVL) and mean free path $(\lambda)$. The results for such parameters at different neutron energies are listed in Tables 5 and 6 respectively. The obtained data indicate a successive improvement in the attenuation properties of neutrons by increasing concentrations of $\mathrm{CdO}$. The results reveal the effectiveness of using the present glass composition in the various applications of neutron shielding.

Table (5): The neutrons half value layers for glass samples under investigation

\begin{tabular}{|c|c|c|c|c|c|}
\hline \multirow{2}{*}{$\begin{array}{l}\text { Neutron } \\
\text { Energy }\end{array}$} & \multicolumn{4}{|c|}{ HVL (cm) } & \multirow{2}{*}{$\begin{array}{c}\text { (HVLS1- }^{\prime} \\
\text { HVLs4\%/HVLs1) } \\
100(\%)\end{array}$} \\
\hline & S1 & S2 & S3 & S4 & \\
\hline Slow & 14.93 & 7.671 & 6.724 & 6.646 & 55.8 \\
\hline Total slow & 11.516 & 9.389 & 8.639 & 8.599 & 25.9 \\
\hline$E>10 \mathrm{keV}$ & 16.746 & 15.194 & 14.704 & 15.526 & 7.6 \\
\hline
\end{tabular}

Table (6): The neutrons mean free paths for glass samples under investigation

\begin{tabular}{lcccc}
\hline \multirow{2}{*}{ Neutron Energy } & \multicolumn{4}{c}{ MFP $(\lambda-\mathbf{c m})$} \\
\cline { 2 - 5 } & S1 & S2 & S3 & S4 \\
\hline Slow & 21.541 & 11.066 & 9.7010 & 9.5870 \\
Total slow & 16.613 & 13.545 & 12.465 & 12.406 \\
E $>\mathbf{1 0}$ keV & 24.160 & 21.920 & 21.214 & 22.399 \\
\hline
\end{tabular}




\subsection{Macroscopic effective removal cross section for fast Neutrons $\Sigma_{R}$}

The macroscopic effective removal cross section $\left(\Sigma_{\mathrm{R}}\right)$ is the probability of a neutron undergoing certain reaction per unit length of moving through the shielding material [36]. The $\Sigma_{R}$ values for the present glass samples have been evaluated at $2 \mathrm{MeV}$ using MCNP5 code and the results are listed in Table 7 . The calculated values of $\Sigma_{R}$ are varied in the range $(0.0813-0.091$ $\mathrm{cm}^{-1}$ ). However, the sample S3 is found to have the highest value of $\Sigma_{R}$. This supports the suggestion that the sample S3 is more effective for fast neutron attenuation than the other samples in the present glass composition.

Table (7): Fast neutrons effective removal cross sections for $\mathrm{CdO}$ containing glasses.

\begin{tabular}{lc}
\hline Sample & $\boldsymbol{\Sigma}_{\mathrm{R}}\left(\mathbf{c m}^{-\mathbf{1}}\right)$ \\
\hline S1 & 0.0813 \\
S2 & 0.0890 \\
S3 & 0.0910 \\
S4 & 0.0900 \\
\hline
\end{tabular}

\section{CONCLUSIONS}

In the present work successful glass series of chemical composition $40 \mathrm{P}_{2} \mathrm{O}_{5}-5 \mathrm{Na}_{2} \mathrm{O}-(35-x) \mathrm{ZnO}-x \mathrm{CdO}-$ $20 \mathrm{BaO}$ where, $(\mathrm{x}=0,7.5,15,22.5 \mathrm{~mol} . \%)$ have been prepared in order to study their shielding characteristics against gamma rays, fast and slow neutrons. The attenuation coefficient $\left(\mu_{\mathrm{m}}\right)$ of the present glass samples have been determined at nine energies of gamma-rays (121.78, 344.27, 661.64, 778.9, 964, 1112.4, 1173.23, $1332.51,1407.92 \mathrm{keV})$. For all glass samples, $\mu_{\mathrm{m}}$ is found to be decreased by increasing the photon energy. At low photon energies (between 121.8 and 344.14 $\mathrm{KeV}), \mu_{\mathrm{m}}$ is decreased dramatically with increasing the photon energy which is attributed to the photoelectric absorption mechanism meanwhile in region from 344.14 $\mathrm{keV}$ up to $1407 \mathrm{keV}, \mu_{\mathrm{m}}$ exhibits a slight decrease which is ascribed to the preeminence of Compton scattering process. The measured values of mass attenuation coefficient for the glass samples at different cadmium concentration are observed to be greatly consistent with those estimated using MCNP5 code and calculated theoretically using WINXCOM software. The half value layers (HVL), mean free path (MFP), effective atomic number $\left(\mathrm{Z}_{\mathrm{eff}}\right)$, effective electron density $\left(\mathrm{N}_{\mathrm{el}}\right)$, and radiation protection efficiency (RPE) have been reported. The role of increasing $\mathrm{CdO}$ is clear and the results reveal the practicality of using the present glass composition as an effective agent for gamma-rays radioprotection.

On the other hand, the macroscopic cross-sections $\left(\Sigma_{\mathrm{n}}\right)$ of the prepared glass samples against slow, total slow and neutrons with energy $>10 \mathrm{keV}$ have been determined under good experimental geometric condition. In case of total slow and slow neutrons, $\Sigma_{\mathrm{n}}$ is increased by increasing content of $\mathrm{CdO}$ from $0 \%$ up to $22.5 \%$ with no significant change between $\mathrm{CdO}$ concentrations from $15 \%$ to $22.5 \%$. In case of neutrons of energy $>10 \mathrm{keV}, \Sigma_{\mathrm{n}}$ is increased by increasing $\mathrm{CdO}$ concentration from $0 \%$ to $15 \%$ then it decreases as $\mathrm{CdO}$ concentration increases to $22.5 \%$. The half value layer (HVL) and relaxation length $(\lambda)$ are also calculated. The macroscopic effective removal cross section $\left(\Sigma_{R}\right)$ for the present glass samples have been evaluated at $2 \mathrm{MeV}$ using MCNP5 code. The calculated values of $\Sigma_{R}$ are varied in the range $\left(0.0813-0.091 \mathrm{~cm}^{-1}\right)$. The data indicate general improvement in the shielding properties of neutrons by increasing concentrations of $\mathrm{CdO}$ with the sample of $15 \%$ cadmium concentration is the optimum percentage in glass barriers, and it is possible to be used as a good neutrons filter. The data obtained in this study should be helpful in potential applications.

\section{REFERENCE}

[1] Parminder Kaur, K.J. Singh, Murat Kurudirek, Sonika Thakur, 2019, Study of environment friendly bismuth incorporated lithium borate glass system for structural, gamma-ray and fast neutron shielding properties, Spectrochimica Acta Part A: Molecular and Biomolecular Spectroscopy, 223, 117309

[2] Murat Kurudirek, 2017Matori, K.A., Sayyed, M.I., Sidek, H.A.A., Zaid, M.H.M., Singh, V.P., 2017. J. Non-Cryst. Solids 457, P.97..

[3] Agar, O. Boztosun, I. Segebade, C., 2017. Multi elemental analysis of some soils in Karaman by PAA using a cLINAC. Appl. Radiat. Isot., 122, P.57-62.

[4] Kavun Y, Boztosun I, Dapo H, MaraşI, Segebade C., 2018. Determination of the $\mathrm{Sr} / \mathrm{Ca}$ ratio of tooth samples by photo activation analysis in Southern Turkey. Radio. Chim. Acta.

[5] Kahraman A, Yilmaz E, Aktag A, Kaya S., 2016. Evaluation of radiation sensor aspects of $\mathrm{Er} 2 \mathrm{O} 3$ MOS capacitors under zero gate bias. IEEE Trans Nucl. Sci., 63. P.1284-93.

[6] Kahraman A, Yilmaz E., 2017. Irradiation response of radio-frequency sputtered Al/ Gd2O3/p-Si MOS capacitors. Radiat. Phys. Chem., 139, P.114-9. 
[7] Wang XB, Wang CN, Zhang YC, Liu TT, Lv JP, Shen X, 2018. Effects of gamma radiation on microbial, physicochemical, and structural properties of whey protein model system. J. Dairy Sci.

[8] Dong M. G. , Sayyed M. I., Lakshminarayana G.,* , Çelikbilek Ersundu M., Ersundu A.E., Priyanka Nayar, Mahdi M.A., 2017, Investigation of gamma radiation shielding properties of lithium zinc bismuth borate glasses using XCOM program and MCNP5 code, Journal of Non-Crystalline Solids, 468 p $12-16$

[9] Obaid SS, Gaikwad DK, Pawar PP., 2018. Determination of gamma ray shielding parameters of rocks and concrete. Radiat. Phys. Chem., 144, P.356-360.

[10] Tuscharoen, S. Kaewkhao, J. Limkitjaroenporn, P. Limsuwan, P. Chewpraditkul, W., 2012. Improvement of $\mathrm{BaO}$ : $\mathrm{B} 2 \mathrm{O} 3$ : Fly ash glasses: radiation shielding, physical and optical properties, Ann. Nucl. Energy 49, P.109-113,

[11] Ashok Kumar, Sayyed M.I., Mengge Dong, Xiangxin Xue, Effect of $\mathrm{PbO}$ on the shielding behavior of $\mathrm{ZnO}-\mathrm{P} 2 \mathrm{O} 5$ glass system using Monte Carlo simulation, 2018, journal of Non-Crystalline Solids, 481, pp. 604-607

[12] Ashok Kumar, Sayyed M.I., Mengge Dong, Xiangxin Xue, 2018, Effect of $\mathrm{PbO}$ on the shielding behavior of ZnO-P2O5 glass system using Monte Carlo simulation, journal of NonCrystalline Solids, 481, pp. 604-607.

[13] Lakshminarayana G., Elmahroug Y., Ashok Kumar, Dong, M.G., Dong-Eun Lee, Jonghun Yoong, Taejoon Park, 2020, TeO2-B2O3-ZnO-La2O3 glasses: $\quad \gamma$-ray and neutron attenuation characteristics analysis by WinXCOM program, MCNP5, Geant4, and Penelope simulation codes, Ceramics International, 46, Pp16620-16635

[14] Agar O., Sayyed M.I., Tekin H.O., Kawa M. Kaky, Baki S.O., Kityk I., 2020, An investigation on shielding properties of $\mathrm{BaO}, \mathrm{MoO} 3$ and $\mathrm{P} 2 \mathrm{O} 5$ based glasses using MCNPX code, 12, pp. 629634

[15] Yasser B. Saddeek, Shams A.M. Issa, Alharbi T., Aly K., Mahmoud Ahmad, Tekine H.O., 2020, Mechanical and nuclear shielding properties of sodium cadmium borate glasses: Impact of cadmium oxide additive, Ceramics International, 46, 2661-2669
[16] Sayyed M.I., Ashok Kumar, Tekind H.O., Ramandeep Kaur, Mandeep Singh, Agarg O., Mayeen Uddin Khandaker, 2020, Evaluation of gamma-ray and neutron shielding features of heavy metals doped $\mathrm{Bi} 2 \mathrm{O} 3-\mathrm{BaO}-\mathrm{Na} 2 \mathrm{O}-\mathrm{MgO}-\mathrm{B} 2 \mathrm{O} 3$ glass systems, Progress in Nuclear Energy, 118.

[17] Reza Bagheri and Ruhollah Adeli, 2020, Gammaray shielding properties of phosphate glasses containing $\mathrm{Bi} 2 \mathrm{O} 3, \mathrm{PbO}$, and $\mathrm{BaO}$ in different rates, Radiation Physics and Chemistry, 174, 108918

[18] Al-Buriahi M.S., Abouhaswa A.S., Tekin H.O., Sriwunkum C., El-Agawany, Nutaro F.I., T., Esra Kavaz, Rammah Y.S., 2020, Structure, optical, gamma-ray and neutron shielding properties of $\mathrm{NiO}$ doped $\mathrm{B} 2 \mathrm{O} 3-\mathrm{BaCO} 3-\mathrm{Li} 2 \mathrm{O} 3$ glass systems, Ceramics International, 46, pp. 1711-1721

[19] Bünyamin Aygün, Erdem Şakar, Esra Cinan, Nergiz Yıldız Yorgun, Sayyed M.I., Agare O., Abdulhalik Karabulut, 2020, Development and production of metal oxide doped glasses for gamma ray and fast neutron shielding, Radiation Physics and Chemistry, 174, 108897

[20] Agar O., Khattari Z.Y., Sayyed M.I., Tekin H.O., Al-Omari S., Maghrabi M., Zaid M.H.M., and Kityk I.V., Evaluation of the shielding parameters of alkaline earth based phosphate glasses using MCNPX code, 2019, 12, Pp. 101-106

[21] Elbashar, YH., Abd El-Ghany, HA., 2017. Optical spectroscopic analysis of $\mathrm{Fe} 2 \mathrm{O} 3$ doped $\mathrm{CuO}$ containing phosphate glass. Opt. Quant. Electron. 49:310, P. 1-13.

[22] Abd El-Ghany, HA., 2018. Characterization and Optical Properties of MnO Doped CuO-Containing Phosphate Glass as Absorption Filters. J. Adv. Phys. 15, P. 5983-5996.

[23] Abd El-Ghany, HA., 2018. Development of a New Glass for Both Visible and Near-Infrared Optical Applications. Key Eng. Mater. 786, P. 224-235.

[24] Haijian Li, Jianhua Yi, Zhao Qin, Zhihua Sun, Yi $\mathrm{Xu}$, Changjian Wang, Fengqi Zhao, Yucheng Hao, Xiaofeng Liang, 2019, Structures, thermal expansion, chemical stability and crystallization behavior of phosphate-based glasses by influence of rare earth, Journal of Non-Crystalline Solids, 522,119602

[25] Grelowska, I. Roben, M. Burtan, B. Sitarz, M. Cisowski, J. Sayed, Y.E. Knapik, A. and Dodek, M., 2016, Structural and optical study of telluritebarium glasses. Journal of Molecular structure, 1126, P. 219-225. 
[26] Maiti, P.K., Mallik, A., Basumajumdar, A. and Guha, P., 2012, "Influence of barium oxide on the crystallization, microstructure and mechanical properties of potassium fluorophlogopite glassceramics", Journal of Ceramics International. 38, P. 251-258.

[27] Agar, O., 2018. Investigation on Gamma Radiation Shielding Behaviour of $\mathrm{CdO}-\mathrm{WO} 3-\mathrm{TeO} 2$ Glasses from 0.015 to $10 \mathrm{MeV}$. Cumhuriyet Sci. J., 39, P.983-990

[28] Erdem Şakar, Özgür Fırat Özpolat, Bünyamin Alım, Sayyed M.I., Murat Kurudirek, Phy-X / PSD: Development of a user friendly online software for calculation of parameters relevant to radiation shielding and dosimetry, 2020, 166, 108496

[29] Hubbell, JH., 1982. Photon mass attenuation and energy-absorption coefficients. Int. J Appl. Radiat. Isot., 33 P.1269-1290.

[30] Sayyed M.I., Tekin H.O., 1lıcoglu O. K, Agar O., Zaid M.H.M., 2018 Shielding features of concrete types containing sepiolite mineral: Comprehensive study on experimental, XCOM and MCNPX results, results in physics, 11, P.40-45

[31] Kumar, A., 2017 Gamma ray shielding properties of $\mathrm{PbO}-\mathrm{Li} 2 \mathrm{O}-\mathrm{B} 2 \mathrm{O} 3$ glasses. Radiat. Phys. Chem., 2017; 136:50-3.
[32] T.Z. Amer, Sara E. Saleh, R. M. El Shazly, N.S. Gomaa, A.A. Bahgat, 2019, Study of the physical and nuclear properties of liquid $\mathrm{PbBiCd}$ alloy coolant in nuclear fast reactor, Journal of Nuclear Materials 522, 226-235.

[33] El Shazly R. M. and Sadawy M.M., Effect of Slag as a Fine Aggregate on Mechanical, Corrosion, and Nuclear Attenuation Properties of Concrete, 2107, International Journal of Scientific Engineering and Research (IJSER), 6, 2347-3878

[34] Akman, F. Geçibesler, IH. Sayyed MI, Tijani, SA. Tufekci, AR. Demirtas, I., 2018. Determination of some useful radiation interaction parameters for waste foods. Nucl. Eng. Technol. 50, P.944-949.

[35] Sayyed MI. and Lakshminarayana, G., 2018. Structural, thermal, optical features and shielding parameters investigations of optical glasses for gamma radiation shielding and defense applications. J. Non Cryst. Solids., 487, P.53-59.

[36] Lakshminarayana, G., Baki, S.O., Lira, A., Kityk, I.V., Caldiño, U., Kaky, K.M., Mahdi, M.A., 2017. Structural, thermal and optical investigations of Dy3+-doped B2O3-WO3-ZnO-Li2O-Na2O glasses for warm white light emitting applications. J. Lumin. 